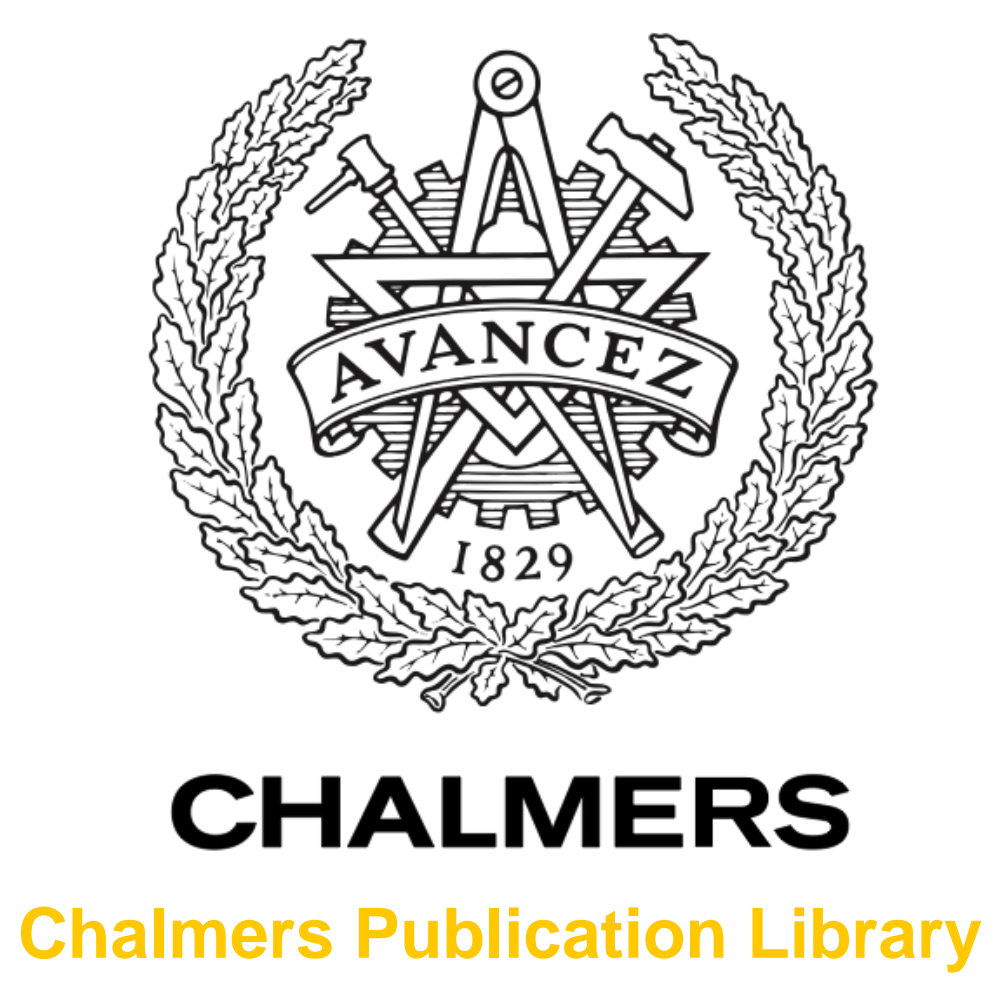

\title{
Analytical modeling and experimental verification of coupling between transmission lines in gap-waveguides
}

This document has been downloaded from Chalmers Publication Library (CPL). It is the author's version of a work that was accepted for publication in:

Proceedings of 6th European Conference on Antennas and Propagation, EuCAP 2012. Prague, 26-30 March 2012

Citation for the published paper:

Bosiljevac, M. ; Sipus, Z. ; Kildal, P. (2012) "Analytical modeling and experimental verification of coupling between transmission lines in gap-waveguides". Proceedings of 6th European Conference on Antennas and Propagation, EuCAP 2012. Prague, 26-30 March 2012 pp. 1402-1406.

http://dx.doi.org/10.1109/EuCAP.2012.6206349

Downloaded from: http://publications.lib.chalmers.se/publication/156677

Notice: Changes introduced as a result of publishing processes such as copy-editing and formatting may not be reflected in this document. For a definitive version of this work, please refer to the published source. Please note that access to the published version might require a subscription. 


\section{LTE MIMO Multiplexing Performance Measured in Reverberation Chamber and Accurate Simple Theory}

\author{
Per-Simon Kildal, Ahmed Hussain, Giuseppe Durisi \\ Department of Signals and Systems \\ Chalmers University of Technology \\ Gothenburg, SWEDEN \\ per-simon.kildal@chalmers.se
}

\author{
Charlie Orlenius, Anton Skårbratt \\ Bluetest AB \\ Gothenburg, SWEDEN
}

\begin{abstract}
We present measurement results of the data-rate throughput inside a reverberation chamber of a commercial USB dongle operating in $2 \times 2$ MIMO spatial multiplexing configuration. A simple and accurate theory is provided by which the measured throughput can be accurately explained. The theory is based on a recently published digital threshold receiver model.
\end{abstract}

\section{INTRODUCTION}

The reverberation chamber is well established as an accurate tool for measuring efficiency, diversity gain and maximum available throughput of mobile terminals equipped with multi-port antennas. The measurement procedures have been extended to total radiated power and receiver sensitivity, and, more recently, to data-rate throughput of WiFi and LTE devices [1].

In order to understand the measurements and to thereby improve the measurement quality, it is important to have a simple theory that can explain the results obtained through measurements. Such a simple theory has recently been presented for the throughput of LTE devices with MIMO and OFDM [2]. The predicted throughput curves, which take into account the spatial and frequency diversity provided by LTE, are within a few tenths of $\mathrm{dB}$ of the measured throughput curves when plotted versus averaged maximum available received signal power. This good agreement is achieved by using the calibrated average power transfer function of the chamber (i.e., the channel path loss), and the threshold of the digital receiver measured directly with a cable between the instrument and the receiver. The theoretical model in [2] is based on approximating the receiver as an ideal digital threshold receiver, which receives all bits without errors in an additive white Gaussian noise (AWGN) channel (i.e., a channel without fading) when the signal power exceeds the threshold value. The purpose of the present paper is to extend this theoretical model to the case when MIMO is used to provide spatial multiplexing. Specifically, we will focus on a $2 \times 2$ MIMO system set up to transmit 2 parallel data streams (instead of providing four independently fading channels), thereby doubling the throughput.

The theoretical model provided in [2] only included $2 \times 1$ MISO and $1 \times 2$ SIMO diversity configurations. At that time, measurements were available also for the case of $2 \times 2$ MIMO used in spatial multiplexing configuration, but the results were left out because we were not able to explain what seemed to be an erroneous or anomalous behavior. However, we have since found a satisfactory physical explanation for our observations, which we have been able to account for using a simple extension in our theoretical model. This new insight contributes to the general understanding of how MIMO systems perform in real-world fading environments.

The main references for a description of how to measure antenna diversity and MIMO system capacity in a reverberation chamber are [3] and [4]. The formulation of the wireless channel as well as the uncertainty can be found in [5], and a summary of all developments till now including active measurements in [6]. The reverberation chamber is characterized by its average mode bandwidth, which is equal to the coherence bandwidth of the wireless channels [7] and can be controlled by loading the chamber. The uncertainty is conveniently described by an average K-factor [8]. The measurements of the wireless channel in the reverberation chamber can be used to determine the total embedded radiation efficiencies on each antenna port, correlation between ports, and diversity gains, as well as maximum available capacity of MIMO antenna systems. The measurement results agree very well with results produced numerically from embedded far field functions measured in anechoic chambers [9]. Thus, the reverberation chambers provide a unique way to determine the performance metrics listed above, without needing to know the radiation patterns.

This work was supported in part by the Swedish Foundation for Strategic Research (SSF) via the CHARMANT antenna systems research center at Chalmers, and by the Swedish Governmental Agency for Innovation Systems (VINNOVA) within the VINN Excellence Center Chase.

\section{SIMPLE THROUGHPUT MODEL FOR MIMO IN SPATIAL MULTIPLEXING CONFIGURATION}

The simple theoretical model for the data-rate throughput proposed in [2] is based on the observation that the group error rate (GER) of modern wireless systems equipped with powerful iterative forward error-correcting codes [10] and operating over a non-fading (static) AWGN channel can be well approximated by the following step function 


$$
\operatorname{GER}_{\text {ideal }}(P)=\left\{\begin{array}{ll}
1 & \text { when } P<P_{t} \\
0 & \text { when } P>P_{t}
\end{array} .\right.
$$

Here, $P$ is the received signal power, and $P_{t}$ is the threshold level delimiting the operating regime of the forward errorcorrecting code. We refer to the receiver having the GER in (1) as ideal digital threshold receiver. Note that GER can refer either to frame error rate (FER), block error rate (BLER) or package error rate (PER). The presence of fading in the wireless communication link can be accounted for by averaging (1) with respect to the fading statistics. For the case of a MIMO-OFDM system in diversity configuration, this averaging yields the following simple formula for the data-rate throughput [2]:

$$
T=\operatorname{Max} * \operatorname{CCDF}\left(P_{t} / P_{a v}\right) \text {. }
$$

Here, CCDF denotes the complementary CDF, CDF the cumulative distribution function of the received power (after diversity combination), $P_{a v}$ is the average received power of a single reference channel in the environment having an antenna with $100 \%$ efficiency, and Max the transmitted data rate of the base station emulator. We see that, for the ideal threshold receiver, the data-rate throughput is proportional to the CCDF of the received power (which can be modeled as a random variable due to fading) at the threshold level.

When MIMO is used in spatial multiplexing configuration, parallel data streams can be transmitted over the wireless fading channel. Interference between the data streams can be removed using suitable pre-processing and post-processing techniques [11]. Hence, from a digital receiver perspective, a $2 \times 2$ MIMO system operating in spatial multiplexing configuration can be described by means of the block diagram given in Figure 1. Referring to this figure let now $P_{t l}$ be the threshold of a digital receiver that operates exclusively over channel 1 . Similarly let $P_{t 2}$ be the threshold of a digital receiver operating exclusively over channel 2. Because the forward error-correcting code operates jointly over the two parallel data streams, the predicted throughput formula (4) needs to be modified as follows:

$$
T=2 * \operatorname{Max} * \operatorname{CCDF}\left(P_{t a v} / P_{a v}\right) .
$$

In essence, $P_{t}$ in (4) needs to be replaced by the arithmetic mean $P_{t a v}=\left(P_{t 1}+P_{t 2}\right) / 2$ of the thresholds associated to the two channels. In other words, from the perspective of a digital threshold receiver, the throughput is twice that of a single data stream, and it is transmitted over a channel with threshold $P_{\text {tav }}$ and same average power. We hasten to add that (5) is derived under the assumption of spatial multiplexing in open loop mode (no channel state information available at the transmitter side). When channel state information is available at the transmitter side and spatial multiplexing operates in closed loop mode, the throughput formula in (5) needs to be modified to account for the possible non-uniform power allocation across the two data streams. We will assume throughout the paper that spatial multiplexing is used in open loop configuration.
It should be noted that taking the arithmetic mean of the thresholds corresponds to making an inverse combination of the power of the two channels. E.g., if channels 1 and 2 are attenuated by efficiency factors $e_{1}$ and $e_{2}$, respectively, the equivalent threshold receiver of each channel degrades from $P_{t 1}=P_{t 2}=P_{t}$ to $P_{t 1}=P_{t} / e_{1}$ and $P_{t 2}=P_{t} / e_{2}$, respectively, so that the average of the two threshold levels become an inverse combination of the channel efficiencies, i.e.,

$$
P_{t a v}=\frac{P_{t 1}+P_{t 2}}{2}=\frac{P_{t}}{2}\left(\frac{1}{e_{1}}+\frac{1}{e_{2}}\right)=\frac{P_{t}}{2} \frac{\left(e_{1}+e_{2}\right)}{e_{1} e_{2}} .
$$

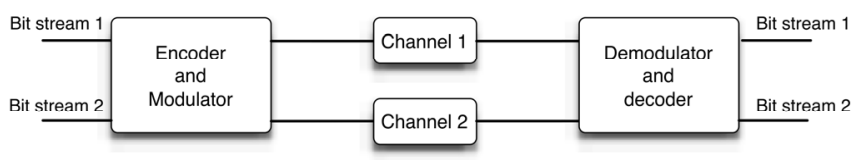

Figure 1. Digital receiver block diagram of a 2x2 MIMO system operating in spatial multiplexing configuration.

The combination in (6) can also be explained in terms of an automatic gain control in connection with each channel receiver, before digitalization. This amplifies the signal and noise of the most attenuated channel. This noise enhancement on the most attenuated channel is responsible for the performance degradation.

\section{Measurements}

We used the measurement setup for data-rate throughput described in [1] to test commercial LTE USB modems with external antennas. The USB modem was located in a separate shielded box outside the reverberation chamber. Thereby, we could connect it with separate antennas located inside the reverberation chamber by means of two cables, and be sure that the modem's built-in antenna did not contribute to the received signal. The RMS delay spread of the reverberation chamber was tuned to 90 ns corresponding to a coherence bandwidth of $3 \mathrm{MHz}$. An R\&S CMW500 was used as base station emulator and measurements were performed on LTE band 7, channel 3100 (2655 MHz). We enforced a 2x2 MIMO spatial multiplexing configuration (open loop), with fixed modulation and bandwidth of 10 $\mathrm{MHz}$, which results in a maximum data-rate throughput of about 40.2 Mbps. Note that there are two cables connected to the base station emulator, which enables measurements with two transmitting antennas.

\section{A. Conductive static measurements}

First we performed conductive measurements, i.e., we measured the data-rate throughput with the two cables from the base station emulator connected directly to the two antenna ports of the LTE device. In this case, we have two parallel channels with constant signal level (no fading). The purpose of the conductive measurements is to determine the threshold to be used in the predicted throughput formula (5). To verify the validity of (6) for the static case in which CCDF reduces to a step function, we connected different attenuators to the antenna ports of the USB dongle.

Both the measured and theoretical results are shown in 
Figure 2 for different port attenuations. The theoretical curves are obtained by approximating the threshold value of the first left curve to $P_{t}=-83 \mathrm{dBm}$, and then by calculating the theoretical threshold values of the others by using (6). We see that all the measured throughput curves resemble those of an ideal threshold receiver with a very steep transition from zero to maximum throughput, and we even see that the shift of the threshold value is accurately described by (6). The performance deteriorates strongly when one port is attenuated in agreement with (6).

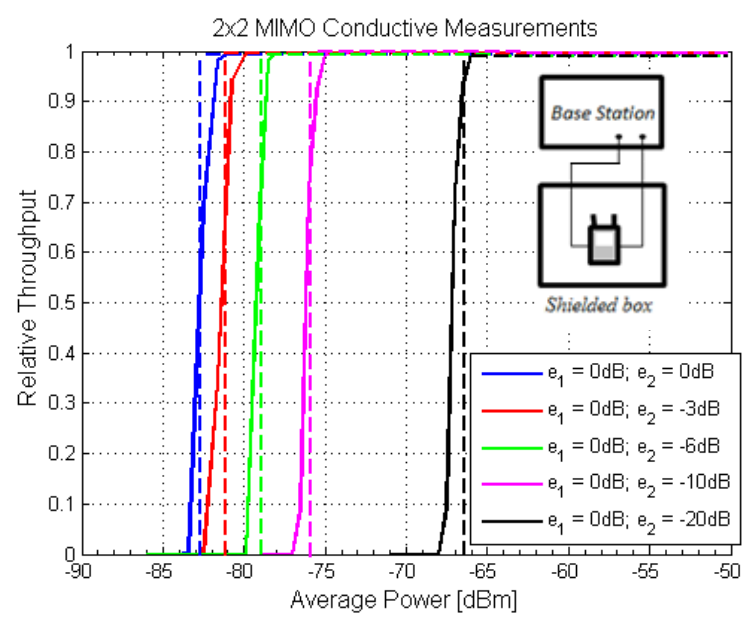

Figure 2. Measured (solid lines) and theoretical (dashed lines) relative data-rate throughput for different values of amplitude imbalance between the two receiving ports of a commercial LTE USB device, for conductive static measurements with an instrument setup corresponding to $2 \times 2 \mathrm{MIMO}$ spatial multiplexing. The measurement setup is shown inside the graph.

\section{B. $\quad$ Dynamic fading using reverberation chamber}

To test the validity of (5)-(6) in the presence of fading, we connected two separate wideband high-efficiency antennas to the cables instead of the base station emulator, and we connected the two ports of the base station emulator to two fixed antennas in the reverberation chamber. We use again $P_{t,}=-83 \mathrm{dBm}$ and (6) to account for the port attenuations, and we also calibrated away the average power transfer function (path loss) of the reverberation chamber and differences in cable losses compared to the static conductive measurement setup, according to normal procedures.

The following MRC diversity combination scheme is used to generate the theoretical CDF of each bit stream channel from random Gaussian power samples:

$$
P_{c}=\frac{1}{4} \frac{1}{N_{f d}} \sum_{i f=1}^{N_{f d}}\left[P_{11, i f}+P_{22, i f}\right] .
$$

Here, $N_{f d}$ is the number of independent frequency subchannels, which depends on the channel coherence bandwidth. We choose $N_{f d}=2$ because the measurements were done under the same conditions as in [2]. The factor 1/4 in front of the equation takes into account the fact that there is no power gain resulting from the use of two transmit antennas, and that the power gain of the two receive antennas is divided between the two bit streams. The additional factor $1 / N_{f d}$ is used to normalize the average path loss to 1 . The curves in Figure 3 are plotted against the calibrated power values. We see that, the theoretical curves obtained using (5)-(6)-(7) match the measurement results well. As expected, the throughput curves no longer have well-defined thresholds due to the presence of Rayleigh fading. As in the conductive static case, performance deteriorates when we reduce the efficiency of the antennas by adding an attenuator at one of the ports.
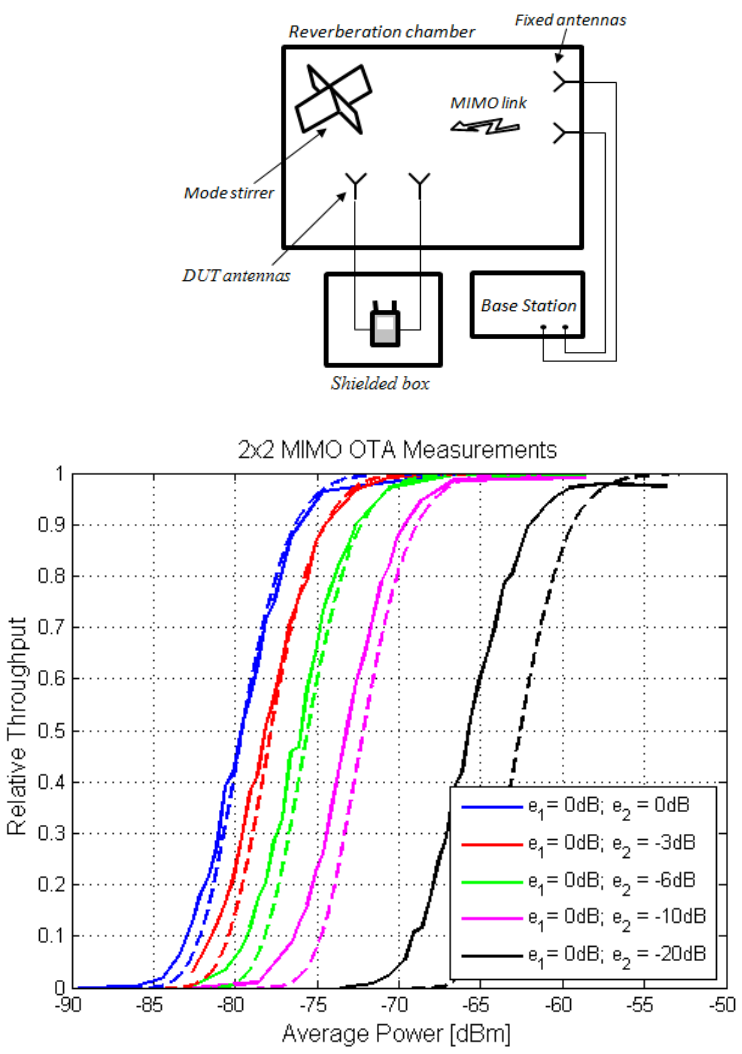

Figure 3. Measured (solid lines) and theoretical (dashed lines) relative data-rate throughput for different values of amplitude imbalance between the two receiving ports of a commercial LTE USB device, for a dynamic Rayleigh-fading case and 2x2 MIMO spatial multiplexing configuration. The measurement setup is shown above the graph.

\section{CONCLUSION}

We have extended the simple theoretical model for the data-rate throughput of LTE systems with OFDM developed in [2] to the case where MIMO is used to provide spatial multiplexing. The model was validated by means of throughput measurements performed in a reverberation chamber using a commercial USB dongle. Both theory and measurement results show significant performance deterioration when attenuators are inserted at one of the antenna ports. We hasten to add that the reported performance deterioration will not be observed in reality: LTE is a dynamic system that automatically selects the best configuration available for the given channel conditions. In 
particular, MIMO spatial multiplexing is not used if the channel conditions do not support it. The new theory improves the quality of OTA measurements, because it allows one to understand the measurement results in detail.

\section{ACKNOWLEDGEMENT}

The authors are thankful for discussions with Buon Kiong Lau about MIMO multiplexing.

\section{REFERENCES}

[1] A. Skårbratt, J. Åsberg, and C. Orlenius, "Over-the-Air Performance Testing of Wireless Terminals by Data Throughput Measurements in Reverberation Chamber," in 2011 European Conference on Antennas and Propagation (EuCAP2011), Rome, 2011.

[2] P. S. Kildal, A. Hussain, X. Chen, C. Orlenius, A. Skårbratt, J. Åsberg, T. Svensson, and T. Eriksson, "Threshold Receiver Model for Throughput of Wireless Devices with MIMO and Frequency Diversity Measured in Reverberation Chamber," IEEE Antennas and Propagation Wireless Letters, vol. 10, pp. 1201-1204, October 2011.

[3] P. S. Kildal and K. Rosengren, "Correlation and capacity of MIMO systems and mutual coupling, radiation efficiency, and diversity gain of their antennas: simulations and measurements in a reverberation chamber," Communications Magazine, IEEE, vol. 42, pp. 104-112, 2004.

[4] K. Rosengren and P. S. Kildal, "Radiation efficiency, correlation, diversity gain and capacity of a six-monopole antenna array for a MIMO system: theory, simulation and measurement in reverberation chamber," Microwaves, Antennas and Propagation, IEE Proceedings , vol. 152, pp. 7-16, 2005.

[5] P.-S. Kildal, X. Chen, C. Orlenius, M. Franzén, and C. Lötbäck Patané, "Characterization of Reverberation Chambers for OTA Measurements of Wireless Devices: Formulation of Channel Matrix and Uncertainty," submitted to IEEE Transactions on Antennas and Propagation, September 2011.

[6] P.-S. Kildal, C. Orlenius, and J. Carlsson, "OTA Measurement Technology Adapted to Antennas and Wireless Devices with MIMO for Use in Multipath Environments with Fading," invited paper submitted to Special issue in IEEE Proceedings, August 2011.

[7] X. Chen, P. S. Kildal, C. Orlenius, and J. Carlsson, "Channel Sounding of Loaded Reverberation Chamber for Over-the-Air Testing of Wireless Devices: Coherence Bandwidth Versus Average Mode Bandwidth and Delay Spread," Antennas and Wireless Propagation Letters, IEEE, vol. 8, pp. 678-681, 2009.

[8] X. Chen, P. S. Kildal, and S. H. Lai, "Estimation of Average Rician KFactor and Average Mode Bandwidth in Loaded Reverberation Chamber," Antennas and Wireless Propagation Letters, IEEE, vol. 10, pp. 1437-1440, 2011.

[9] X. Chen, P. S. Kildal, J. Carlsson, and J. Yang, "Comparison of Ergodic Capacities From Wideband MIMO Antenna Measurements in Reverberation Chamber and Anechoic Chamber," Antennas and Wireless Propagation Letters, IEEE, vol. 10, pp. 446-449, 2011.

[10]T. J. Richardson and R. Urbanke, Modern Coding Theory. Cambridge, U.K.: Cambridge Univ. Press, 2008.

[11]D. N. C. Tse and P. Viswanath, Fundamentals of Wireless Communications. Cambridge, U.K.: Cambridge Univ. Press, 2005. 